\title{
Symbol Message Passing Decoding of LDPC Codes for Orthogonal Modulations
}

\author{
Emna Ben Yacoub ${ }^{\ddagger}$, Balazs Matuz ${ }^{\dagger}$ \\ ${ }^{\ddagger}$ Institute for Communications Engineering, Technical University of Munich, Germany \\ ${ }^{\dagger}$ Institute of Communications and Navigation, German Aerospace Center (DLR), Germany \\ Email: emna.ben-yacoub@tum.de,balazs.matuz@dlr.de
}

\begin{abstract}
A simple decoder for $q$-ary low-density parity-check codes is studied, termed symbol message passing. The decoder passes hard decisions from a $q$-ary alphabet. For orthogonal modulations over the additive white Gaussian channel for which the modulation order and the field order $q$ are equal, it is shown that the extrinsic messages can be modelled as observations of a $q$-ary symmetric channel, allowing to work out density evolution equations. A stability analysis is provided which emphasizes the influence of degree- 3 variable nodes. Simulation results show performance gains for increasing $q$ w.r.t. binary low-density parity-check codes with bit-interleaved coded modulation, and potential savings in decoding complexity.
\end{abstract}

\section{INTRODUCTION}

Non-binary low-density parity-check (LDPC) codes have been proposed for various applications to improve the performance of their binary counterparts [1], [2]. Several decoding algorithms have been studied in the literature to decrease the complexity of the non-binary operations in the decoder [3], [4], [5], [6]. Amongst others, Hadamard-Walsh transform based decoders facilitate the computation of the check node $(\mathrm{CN})$ operations [6] while extended min-sum decoders consider only a subset of the $q$ reliabilities of each symbol [3].

Recently, a symbol message passing (SMP) decoder for non-binary LDPC codes over the $q$-ary symmetric channel $(q \mathrm{SC})$ was proposed [7]. Messages in the decoder consist of the most reliable symbol only. This is similar to an extended min-sum decoder where only the most reliable value of a $q$-ary symbol is considered. However, instead of passing reliabilities, hard decisions are propagated in SMP decoders. The simple decoding operations let SMP decoders be used for applications requiring high throughput/low decoding complexity, rather than capacity approaching performance. This stands in contrast to the original purpose of non-binary LDPC codes and decoders.

This work extends the results of [7] on $q \mathrm{SCs}$ to additive white Gaussian noise (AWGN) channels with orthogonal modulations where the field order $q$ and the modulation order are equal. This makes non-binary LDPC codes a natural choice since each $q$-ary modulation symbol is in one-to-one correspondence with a $q$-ary code symbol. Our aim is to show that non-binary LDPC codes with low-complexity decoding algorithms are favorable for certain coded-modulation scenarios. To this end, we provide a density evolution (DE) analysis for orthogonal modulations which finds good degree distributions for unstructured LDPC code ensembles. This is complemented by a stability analysis which shows that not only degree-2, but also degree-3 variable nodes (VNs) must be handled with care. The simple decoding algorithm thresholds show a gap of around $2.1 \mathrm{~dB}$ with respect to channel capacity. However, numerical simulation results show that $q$-ary LDPC codes with SMP decoders outperform binary LDPC codes in a bitinterleaved coded modulation (BICM) setting for increasing $q$, with potentially lower decoding complexity.

\section{PRELIMINARIES}

\section{A. System Model}

Consider LDPC codes over $\mathbb{F}_{q}=\left\{0,1, \alpha, \ldots, \alpha^{q-2}\right\}$ with $q=2^{m}, m$ a positive integer and $\alpha$ a primitive element of $\mathbb{F}_{q}$. We denote a length- $N$ codeword as $\boldsymbol{c}=\left(c_{1}, c_{2}, \ldots, c_{N}\right)$, with $c_{i} \in \mathbb{F}_{q}$. We consider one-to-one mappings between codeword symbols $c_{i}$ and orthogonal modulation symbols $\chi\left(c_{i}\right)$. Each modulation symbol is represented as a length$q$ vector $\chi\left(c_{i}\right)=\left(\chi_{0}\left(c_{i}\right), \chi_{1}\left(c_{i}\right), \ldots, \chi_{\alpha^{q-2}}\left(c_{i}\right)\right)$ where for convenience we index its entries by elements of $\mathbb{F}_{q}$ rather than by integers $1,2, \ldots, q$. For orthogonal modulations $\forall a, a^{\prime} \in \mathbb{F}_{q}$ we have

$$
\left\langle\chi(a), \chi\left(a^{\prime}\right)\right\rangle= \begin{cases}1 & a=a^{\prime} \\ 0 & \text { otherwise }\end{cases}
$$

where $\langle\cdot, \cdot\rangle$ is the inner product. Without loss of generality we consider pulse position modulation (PPM) for which $\forall a \in \mathbb{F}_{q}$

$$
\chi_{a}\left(c_{i}\right)= \begin{cases}1 & a=c_{i} \\ 0 & \text { otherwise }\end{cases}
$$

For transmission over an AWGN channel with input alphabet $\mathcal{X}=\left\{\chi(0), \chi(1), \ldots, \chi\left(\alpha^{q-2}\right)\right\}$, the observation of the $i$ th modulated symbol is

$$
\boldsymbol{y}_{i}=\chi\left(c_{i}\right)+\boldsymbol{n}_{i}
$$

where $\boldsymbol{n}_{i}=\left(n_{i, 0}, n_{i, 1}, \ldots, n_{i, \alpha^{q-2}}\right)$ is the length- $q$ noise vector sampled from $q$ independent and identically distributed Gaussian random variables (RVs) with zero-mean and variance $\sigma_{\mathrm{N}}^{2}$. For ease of notation, we will drop the modulation 
symbol index $i$ whenever possible. For $a \in \mathbb{F}_{q}$ the likelihood $p_{\boldsymbol{Y} \mid A}(\boldsymbol{y} \mid a)$ can be written as

$$
\begin{aligned}
p(\boldsymbol{y} \mid a) & =\left(\frac{1}{\sqrt{2 \pi \sigma_{\mathrm{N}}^{2}}}\right)^{q} \exp \left(-\frac{\|\boldsymbol{y}-\boldsymbol{\chi}(a)\|^{2}}{2 \sigma_{\mathrm{N}}^{2}}\right) \\
& \propto \exp \left(-\frac{1}{\sigma_{\mathrm{N}}^{2}}\langle\boldsymbol{y}, \boldsymbol{\chi}(a)\rangle\right) .
\end{aligned}
$$

Let $E_{\mathrm{b}}$ denote the energy per information bit, $E_{\mathrm{s}}$ the energy per modulation symbol and $N_{0}$ is the one-sided noise power spectral density. Then, we have

$$
\frac{E_{\mathrm{b}}}{N_{0}}=\frac{1}{R m} \frac{E_{\mathrm{s}}}{N_{0}}=\frac{1}{R m} \frac{1}{2 \sigma_{\mathrm{N}}^{2}} .
$$

\section{B. Decoder messages}

The messages in an iterative decoder can be modelled as an observation from an extrinsic channel [8]. For the AWGN channel with $q$-ary orthogonal modulations and SMP decoding of $q$-ary LDPC codes, the extrinsic channel is a $q \mathrm{SC}$. Details follow in Section IV. The transition probabilities of a $q \mathrm{SC}$ with error probability $\epsilon$ are

$$
P(w \mid a)= \begin{cases}1-\epsilon & \text { if } w=a \\ \frac{\epsilon}{q-1} & \text { otherwise. }\end{cases}
$$

For convenience we work with log-likelihood values ( $L$ values) $L_{a}(w)=\log (P(w \mid a)) a \in \mathbb{F}_{q} . L$-values can be compactly expressed as length- $q$ log-likelihood vectors $(\boldsymbol{L}$ vectors) with

$$
\boldsymbol{L}(w)=\left(L_{0}(w), L_{1}(w), \ldots, L_{\alpha^{q-2}}(w)\right) .
$$

Lemma 1. Suppose the $w^{(i)}, i=1,2, \ldots, d$, are observations of a qSC with $1-\epsilon>\frac{\epsilon}{q-1}$. When summing $d \boldsymbol{L}$-vectors $\boldsymbol{L}\left(w^{(i)}\right)$, the elements of the sum with indices $\mathcal{I}=\bigcup_{i=1}^{d} w^{(i)}$, where $|\mathcal{I}| \leq \min (d, q)$, all have values greater than $d \log \frac{\epsilon}{q-1}$ and thus contain the maximum value of the sum.

Proof. Observe that each $\boldsymbol{L}\left(w^{(i)}\right)$ has a single maximum with index $a=w^{(i)}$ and that all other entries are $\log \frac{\epsilon}{q-1}$.

Similarly, let the channel message be represented by a length- $q \boldsymbol{L}$-vector

$$
\boldsymbol{L}(\boldsymbol{y})=\left(L_{0}(\boldsymbol{y}), L_{1}(\boldsymbol{y}), \ldots, L_{\alpha^{q-2}}(\boldsymbol{y})\right)
$$

with $L_{a}(\boldsymbol{y})=\log (p(\boldsymbol{y} \mid a))$. While the transition probability densities $p(\boldsymbol{y} \mid a)$ of the communication channel are given in (1), the transition probabilities of the extrinsic channel are in general unknown, but accurate estimates can be obtained via DE analysis [9].

\section{Non-binary LDPC Codes}

Non-binary LDPC codes can be defined by an $M \times N$ sparse parity-check matrix $\boldsymbol{H}=\left[h_{i, j}\right]$ with entries in $\mathbb{F}_{q}$. The parity-check matrix can be represented by a Tanner graph with $N$ VNs corresponding to codeword symbols and $M \mathrm{CNs}$ corresponding to parity checks. Each edge connecting $\mathrm{v}$ and $\mathrm{c}$ is labeled by a non-zero element $h_{\mathrm{v}, \mathrm{c}}$ of $\boldsymbol{H}$. The sets $\mathcal{N}(\mathrm{v})$ and
$\mathcal{N}(\mathrm{c})$ denote the neighbors of $\mathrm{VN} \mathrm{v}$ and $\mathrm{CN}$ c, respectively. The degree of a VN $\mathrm{v}$ is the cardinality of the set $\mathcal{N}(\mathrm{v})$. Similarly, the degree of a $\mathrm{CN} c$ is the cardinality of the set $\mathcal{N}(\mathrm{c})$. The $\mathrm{VN}$ edge-oriented degree distribution polynomial is $\lambda(x)=\sum_{i=1}^{d_{\mathrm{v}}} \lambda_{i} x^{i-1}$ where $\lambda_{i}$ is the fraction of edges incident to VNs with degree $i$ and $d_{\mathrm{v}}$ is the maximum $\mathrm{VN}$ degree. Similarly, the $\mathrm{CN}$ edge-oriented degree distribution polynomial is $\rho(x)=\sum_{i=2}^{d_{c}} \rho_{i} x^{i-1}$ where $\rho_{i}$ is the fraction of edges incident to CNs with degree $i$ and $d_{\mathrm{c}}$ is the maximum $\mathrm{CN}$ degree. An unstructured irregular LDPC code ensemble $\mathscr{C}_{\lambda, \rho}^{q, N}$ is the set of all $q$-ary LDPC codes with block length $N$ and degree distribution polynomials $\lambda(x)$ and $\rho(x)$.

\section{Symbol Message Passing Algorithm}

Let $m_{\mathrm{c} \rightarrow \mathrm{v}}^{(\ell)}$ be the message sent from $\mathrm{CN} c$ to its neighboring $\mathrm{VN} v$ at the $\ell$ th iteration and $m_{\mathrm{v} \rightarrow \mathrm{c}}^{(\ell)}$ the message sent from $\mathrm{VN} v$ to $\mathrm{CN} c$ at the $\ell$ th iteration. All exchanged messages between the CNs and VNs are from a $q$-ary alphabet. Decoding proceeds as follows.

1) Initialization: Each $\mathrm{VN}$ computes the channel $\boldsymbol{L}$-vector defined in (2) and sends the symbol with the highest $L$-value. Since

$$
L_{a}(\boldsymbol{y})=\frac{y_{a}}{\sigma_{\mathrm{N}}^{2}}-\frac{q}{2} \log \left(2 \pi \sigma_{\mathrm{N}}^{2}\right)-\frac{\|\boldsymbol{y}\|^{2}+1}{2 \sigma_{\mathrm{N}}^{2}} \quad \forall a \in \mathbb{F}_{q}
$$

finding the maximum of the length- $q$ vector $\boldsymbol{L}(\boldsymbol{y})$ is equivalent to finding the maximum of $\boldsymbol{y}$. Hence, we have

$$
m_{\mathrm{v} \rightarrow \mathrm{c}}^{(0)}=\underset{a \in \mathbb{F}_{q}}{\operatorname{argmax}} L_{a}(\boldsymbol{y})=\underset{a \in \mathbb{F}_{q}}{\operatorname{argmax}} y_{a} .
$$

The complexity of the initialization step scales as $\mathcal{O}(N q)$. The performed operation is finding a maximum.

2) $C N$ update: At the $\ell$ th iteration, $\mathrm{CN}$ c sends to its neighboring $\mathrm{VN} v$ the following message:

$$
m_{\mathrm{c} \rightarrow \mathrm{v}}^{(\ell)}=-h_{\mathrm{c}, \mathrm{v}}^{-1} \sum_{\mathrm{v}^{\prime} \in \mathcal{N}(\mathrm{c}) \backslash \mathrm{v}} h_{\mathrm{v}, \mathrm{c}^{\prime}} m_{\mathrm{v}^{\prime} \rightarrow \mathrm{c}}^{(\ell-1)}
$$

where $h_{\mathrm{c}, \mathrm{v}}$ is the respective parity-check matrix element and $h_{\mathrm{c}, \mathrm{v}}^{-1}$ is its inverse in $\mathbb{F}_{q}$. From [7] the $\mathrm{CN}$ operation can be implemented with $2 d_{\mathrm{c}}-1 q$-ary additions and $2 d_{\mathrm{c}} q$-ary multiplications. If $q$-ary additions/multiplications are implemented using elementary operations the complexity may depend on $q$. For instance, the sum of two $q$-ary symbols can be performed by $\log _{2} q$ binary XOR operations. Thus, the complexity scales as $\mathcal{O}\left(M d_{\mathrm{c}} f(q)\right)$, where $f(\cdot)$ is an implementation dependent cost.

3) VN update: Each VN performs the following operations

$$
m_{\mathrm{v} \rightarrow \mathrm{c}}^{(\ell)}=\underset{a \in \mathbb{F}_{q}}{\operatorname{argmax}} L_{\mathrm{ex}, a}^{(\ell)}
$$

where

$$
\begin{aligned}
\boldsymbol{L}_{\mathrm{ex}}^{(\ell)} & =\left[L_{\mathrm{ex}, 0}^{(\ell)}, L_{\mathrm{ex}, 1}^{(\ell)}, \ldots, L_{\mathrm{ex}, \alpha^{q-2}}^{(\ell)}\right] \\
& =\boldsymbol{L}(\boldsymbol{y})+\sum_{\mathrm{c}^{\prime} \in \mathcal{N}(\mathrm{v}) \backslash \mathrm{c}} \boldsymbol{L}\left(m_{\mathrm{c}^{\prime} \rightarrow \mathrm{v}}^{(\ell)}\right) .
\end{aligned}
$$


The complexity of the VN operation scales as $\mathcal{O}\left(N d_{\mathrm{v}}\right)$ : as shown in [7] all $d_{\mathrm{v}}$ extrinsic messages can be computed efficiently from the sum of all incoming messages $\sum_{\mathrm{c}^{\prime} \in \mathcal{N}(\mathrm{v})} \boldsymbol{L}\left(m_{\mathrm{c}^{\prime} \rightarrow \mathrm{v}}^{(\ell)}\right)$ and $\boldsymbol{L}(\boldsymbol{y})$, denoted by $\boldsymbol{L}_{\mathrm{tot}}^{(\ell)}$. Let the entry with index $a$ be the maximum of $\boldsymbol{L}(\boldsymbol{y})$ computed in (3). ${ }^{1}$ By Lemma 1 the largest values of $\boldsymbol{L}_{\text {tot }}^{(\ell)}$ will be in $\mathcal{I} \cup a$, where $|\mathcal{I} \cup a| \leq \min \left(d_{\mathrm{v}}+1, q\right) \leq d_{\mathrm{v}}+1$. This step requires $\mathcal{O}\left(d_{\mathrm{v}}\right)$ additions of floats. The identification of the (two) largest values of $\boldsymbol{L}_{\mathrm{tot}}^{(\ell)}$ requires $d_{\mathrm{v}}$ steps. Then, the extrinsic messages and their maximum can be obtained from $\boldsymbol{L}_{\mathrm{tot}}^{(\ell)}$ with $d_{\mathrm{v}}$ additional operations (subtractions, comparisons) and we have an overall complexity scaling of $\mathcal{O}\left(N d_{\mathrm{v}}\right)$.

4) Hard decision: Each VN computes an estimation of its codeword symbol as follows

$$
\hat{c}_{\mathrm{v}}^{(\ell)}=\underset{a \in \mathbb{F}_{q}}{\operatorname{argmax}}\left(L_{a}(\boldsymbol{y})+\sum_{\mathrm{c}^{\prime} \in \mathcal{N}(\mathrm{v})} L_{a}\left(m_{\mathrm{c}^{\prime} \rightarrow \mathrm{v}}^{(\ell)}\right)\right) .
$$

\section{Density Evolution Analysis}

We discuss DE analysis for SMP for non-binary irregular LDPC code ensembles. Due to symmetry, we can assume the all-zero codeword was transmitted. Let $p_{a}^{(\ell)}\left(s_{a}^{(\ell)}\right)$ be the probability that a variable to check (check to variable) message takes value $a \in \mathbb{F}_{q}$ at the $\ell$ th iteration. Let $\ell_{\max }$ be the maximum number of iterations. In the limit of $N \rightarrow \infty$, the evolution of the message distributions is as follows.

1) Initialization: Define the random vector

$$
\boldsymbol{Z}_{a}=Y_{a} \mathbf{1}_{q-1}-\boldsymbol{Y}_{[a]}
$$

for $a \in \mathbb{F}_{q}$, with $\boldsymbol{Y}_{[a]}$ being the random vector $\boldsymbol{Y}$ of channel observations without the entry $Y_{a}$ and $\mathbf{1}_{q-1}$ the length- $(q-1)$ all-one vector. It is easy to check that $\forall a \in \mathbb{F}_{q}$

$$
p_{a}^{(0)}=\operatorname{Pr}\left\{\boldsymbol{Z}_{a}>\mathbf{0}\right\} .
$$

Conditioned on the transmission of the all-zero codeword, we have $\boldsymbol{Y}$ is a Gaussian random vector with mean $\boldsymbol{\mu}_{\boldsymbol{Y}}=$ $(1,0, \ldots, 0)$ and covariance matrix $\boldsymbol{\Sigma}_{\boldsymbol{Y}}=\sigma_{\mathrm{N}}^{2} \boldsymbol{I}_{q}$, where $\boldsymbol{I}_{q}$ is the size $q$ identity matrix. Thus, $\boldsymbol{Z}_{a}$ is a Gaussian random vector with mean

$$
\boldsymbol{\mu}_{\boldsymbol{Z}_{a}}= \begin{cases}\mathbf{1}_{q-1} & a=0 \\ (-1,0, \ldots, 0) & a \in \mathbb{F}_{q} \backslash\{0\}\end{cases}
$$

and covariance matrix $\boldsymbol{\Sigma}_{\boldsymbol{Z}_{a}}$ with entries

$$
\left(\boldsymbol{\Sigma}_{\boldsymbol{Z}_{a}}\right)_{i, j}= \begin{cases}2 \sigma_{\mathrm{N}}^{2} & i=j \\ \sigma_{\mathrm{N}}^{2} & \text { otherwise. }\end{cases}
$$

The parameters of $\boldsymbol{Z}_{a} \forall a \in \mathbb{F}_{q} \backslash\{0\}$ do not depend on $a$ and thus take the same value. Therefore, $\forall a \in \mathbb{F}_{q} \backslash\{0\}$ we have

$$
p_{a}^{(0)}=\frac{1-p_{0}^{(0)}}{q-1}
$$

\footnotetext{
${ }^{1}$ For an unquantized AWGN channel $\boldsymbol{L}(\boldsymbol{y})$ has a unique maximum with probability one.
}

where

$$
p_{0}^{(0)}=\operatorname{Pr}\left\{\boldsymbol{Z}_{0}>\mathbf{0}\right\}
$$

from (6). Observe that $p_{0}^{(0)}$ and $p_{a}^{(0)}$ with $a \in \mathbb{F}_{q} \backslash\{0\}$ are transition probabilities of a $q \mathrm{SC}$ where $1-p_{0}^{(0)}$ is the channel error probability formerly denoted by $\epsilon$.

2) Evolution of densities for $\ell=1,2, \ldots, \ell_{\max }$ : For the check to variable update we have

$$
s_{0}^{(\ell)}=\frac{1}{q}\left[1+(q-1) \rho\left(\frac{q \cdot p_{0}^{(\ell-1)}-1}{q-1}\right)\right]
$$

and $\forall a \in \mathbb{F}_{q} \backslash\{0\}$

$$
s_{a}^{(\ell)}=\frac{1-s_{0}^{(\ell)}}{q-1} .
$$

Again, the extrinsic channel is a $q \mathrm{SC}$ with error probability $1-s_{0}^{(\ell)}$.

For the variable to check update we introduce the random vector $\boldsymbol{F}^{(\ell)}=\left(F_{0}^{(\ell)}, \ldots, F_{\alpha^{q-2}}^{(\ell)}\right)$, where $F_{a}^{(\ell)}$ denotes the $\mathrm{RV}$ associated to the number of incoming $\mathrm{CN}$ messages to a degree- $d$ VN that takes value $a \in \mathbb{F}_{q}$ at the $\ell$ th iteration, and $f_{a}^{(\ell)}$ is its realization. The entries of $\boldsymbol{L}_{\mathrm{ex}}^{(\ell)}$ in (4) are

$$
\begin{aligned}
L_{\mathrm{ex}, a}^{(\ell)} & =\frac{y_{a}}{\sigma_{\mathrm{N}}^{2}}+\mathrm{D}^{(\ell)} f_{a}^{(\ell)}+K \\
\mathrm{D}^{(\ell)} & =\log \left(s_{0}^{(\ell)}\right)-\log \left(\left(1-s_{0}^{(\ell)}\right) /(q-1)\right) \\
K= & -\frac{q}{2} \log \left(2 \pi \sigma_{\mathrm{N}}^{2}\right)-\frac{\|\boldsymbol{y}\|^{2}+1}{2 \sigma_{\mathrm{N}}^{2}} \\
& +(d-1) \log \left(\left(1-s_{0}^{(\ell)}\right) /(q-1)\right) .
\end{aligned}
$$

$K$ in (7) is independent of $a$ and can be ignored when computing $\boldsymbol{L}_{\mathrm{ex}}^{(\ell)}$. We obtain (8) $\forall a \in \mathbb{F}_{q}$ where $\boldsymbol{f}_{[a]}^{(\ell)}$ is the vector $f^{(\ell)}$ without its entry $f_{a}^{(\ell)}, Z_{a}$ is defined in (5) and the sum is over integer vectors $f^{(\ell)}$ for which it holds $0 \leq f_{u}^{(\ell)} \leq d-1 \forall u \in \mathbb{F}_{q}$ and $\sum_{u \in \mathbb{F}_{q}} f_{u}^{(\ell)}=d-1$ and

$$
\begin{aligned}
\operatorname{Pr}\left\{\boldsymbol{F}^{(\ell)}=\boldsymbol{f}^{(\ell)}\right\}= & \left(\begin{array}{c}
d-1 \\
f_{0}^{(\ell)}, \ldots, f_{\alpha^{q-2}}^{(\ell)}
\end{array}\right)\left(s_{0}^{(\ell)}\right)^{f_{0}^{(\ell)} \times} \\
& \left(\frac{1-s_{0}^{(\ell)}}{q-1}\right)^{d-1-f_{0}^{(\ell)}} .
\end{aligned}
$$

The ensemble iterative decoding threshold $\left(E_{\mathrm{b}} / N_{0}\right)^{\star}$ is defined as the minimum $E_{\mathrm{b}} / N_{0}$ for which $p_{0}^{(\ell)} \rightarrow 1$ as $\ell \rightarrow \infty$.

\section{Stability Condition}

We define $\bar{p}_{0}^{(\ell)}=1-p_{0}^{(\ell)}$ and $\bar{s}_{0}^{(\ell)}=1-s_{0}^{(\ell)}$. The stability analysis examines the convergence of the probability $\bar{p}_{0}^{(\ell)}$ to 


$$
\begin{aligned}
p_{a}^{(\ell)} & =\sum_{d} \lambda_{d} \sum_{\boldsymbol{f}^{(\ell)}} \operatorname{Pr}\left\{\boldsymbol{F}^{(\ell)}=\boldsymbol{f}^{(\ell)}\right\} \operatorname{Pr}\left\{\underset{u \in \mathbb{F}_{q}}{\operatorname{argmax}} L_{\mathrm{ex}, a}^{(\ell)}=a \mid \boldsymbol{f}^{(\ell)}\right\} \\
& =\sum_{d} \lambda_{d} \sum_{\boldsymbol{f}^{(\ell)}} \operatorname{Pr}\left\{\boldsymbol{F}^{(\ell)}=\boldsymbol{f}^{(\ell)}\right\} \operatorname{Pr}\left\{\boldsymbol{Z}_{a}>\sigma_{\mathrm{N}}^{2} \mathrm{D}^{(\ell)}\left(\boldsymbol{f}_{[a]}^{(\ell)}-f_{a}^{(\ell)} \mathbf{1}_{q-1}\right)\right\}
\end{aligned}
$$

zero under the assumption that it is close to the fixed point $\bar{p}_{0}^{\star}=0$. Note that $\bar{s}_{0}^{(\ell)} \rightarrow 0$ as $\bar{p}_{0}^{(\ell)} \rightarrow 0$. Thus, $\mathrm{D}^{(\ell)} \rightarrow \infty$ and

$$
\begin{aligned}
\bar{p}_{0}^{(\ell)}= & \sum_{d} \lambda_{d} \sum_{a \in \mathbb{F}_{q}}\left[\sum_{\boldsymbol{f}^{(\ell)} \in \mathcal{F}_{1, a}} \operatorname{Pr}\left\{\boldsymbol{F}^{(\ell)}=\boldsymbol{f}^{(\ell)}\right\}\right. \\
& \left.+\sum_{\boldsymbol{f}^{(\ell)} \in \mathcal{F}_{2, a}} \operatorname{Pr}\left\{\boldsymbol{F}^{(\ell)}=\boldsymbol{f}^{(\ell)}\right\} \operatorname{Pr}\left\{\underset{e \in \mathcal{S}}{\operatorname{argmax}} Y_{e}=a\right\}\right]
\end{aligned}
$$

where $\mathcal{F}_{1, a}$ is the set of all integer vectors $\boldsymbol{f}^{(\ell)}$ for which it holds $\sum_{u \in \mathbb{F}_{q}} f_{u}^{(\ell)}=d-1$ and $f_{a}^{(\ell)}>f_{u}^{(\ell)} \geq 0 \forall u \in \mathbb{F}_{q} \backslash\{a\}$. $\mathcal{F}_{2, a}$ is the set of all integer vectors $\boldsymbol{f}^{(\ell)}$ for which it holds $\sum_{u \in \mathbb{F}_{q}} f_{u}^{(\ell)}=d-1$ and $f_{a}^{(\ell)}>f_{u}^{(\ell)} \geq 0 \forall u \in \mathbb{F}_{q} \backslash \mathcal{S}_{a}$ where

$$
\mathcal{S}_{a}=\left\{b \in \mathbb{F}_{q} \mid f_{b}^{(\ell)}=f_{a}^{(\ell)}\right\}
$$

and $\left|\mathcal{S}_{a}\right|>1$. Recall that for any $a \in \mathbb{F}_{q} \backslash\{0\}$

$$
\begin{aligned}
\operatorname{Pr}\left\{\boldsymbol{F}^{(\ell)}=\boldsymbol{f}^{(\ell)}\right\}= & \left(\begin{array}{c}
d-1 \\
f_{0}^{(\ell)}, \ldots, f_{\alpha^{q-2}}^{(\ell)}
\end{array}\right)\left(1-\bar{s}_{0}^{(\ell)}\right)^{f_{0}^{(\ell)} \times} \\
& \left(\frac{\bar{s}_{0}^{(\ell)}}{q-1}\right)^{d-1-f_{0}^{(\ell)}} .
\end{aligned}
$$

We obtain

$$
\lim _{\bar{s}_{0}^{(\ell)} \rightarrow 0} \frac{\mathrm{d} \bar{p}_{0}^{(\ell)}}{\mathrm{d} \bar{s}_{0}^{(\ell)}}=\lambda_{2}+2 \lambda_{3} Q\left(\frac{1}{\sqrt{2 \sigma_{\mathrm{N}}^{2}}}\right) .
$$

Furthermore, we have

$$
\bar{s}_{0}^{(\ell)}=\frac{q-1}{q}\left[1-\rho\left(1-\frac{q \bar{p}_{0}^{(\ell-1)}}{q-1}\right)\right]
$$

and

$$
\lim _{\bar{p}_{0}^{(\ell-1)} \rightarrow 0} \frac{\mathrm{d} \bar{s}_{0}^{(\ell)}}{\bar{p}_{0}^{(\ell-1)}}=\rho^{\prime}(1) .
$$

The first order Taylor expansions via (9), (10) yield

$$
\bar{p}_{0}^{(\ell)}=\rho^{\prime}(1)\left[\lambda_{2}+2 \lambda_{3} Q\left(\frac{1}{\sqrt{2 \sigma_{\mathrm{N}}^{2}}}\right)\right] \bar{p}_{0}^{(\ell-1)} .
$$

The stability condition is fulfilled if and only if

$$
\rho^{\prime}(1)\left[\lambda_{2}+2 \lambda_{3} Q\left(\frac{1}{\sqrt{2 \sigma_{\mathrm{N}}^{2}}}\right)\right]<1 .
$$

Remark 1. The fraction of edges connected to degree 2 and 3 VNs impacts the stability condition for SMP decoding. Thus, certain degree distributions optimized for unquantized belief propagation (see, e.g., [10]) might be unsuitable for SMP due to their large number of degree 2 and $3 \mathrm{VNs}$.

\section{NUMERICAL RESULTS}

\section{A. Iterative Decoding Thresholds}

The DE analysis in Section IV suggests an optimization algorithm to find rate $R=1 / 2$ irregular LDPC ensembles with 'good' thresholds for $q \in\{8,16,32\}$. We restrict the maximum VN degree to 12 and perform two optimizations: one without further constraints and one with constraints on the degree two and three VNs. Threshold results are depicted in Table I and show a gap of at least $2.1 \mathrm{~dB}$ with respect to the Shannon limit for various $q$. Thresholds of $q$-ary LDPC codes under full belief propagation (BP) decoding in [10] show only gaps of $0.2 \mathrm{~dB}$, i.e., the simple SMP decoder yields a loss of around $1.9 \mathrm{~dB}$. Interestingly, for binary LDPC codes with orthogonal modulations and BICM (no iterative detection) the gap to coded modulation capacity is comparable or even larger. For instance, for $q=16$ the gap is $1.8 \mathrm{~dB}$ [10, Fig. 1].

\section{B. Monte Carlo Simulations}

We designed three codes with $q \in\{8,16,32\}, N=10000$ (in $\mathbb{F}_{q}$ symbols), and $R=1 / 2$ based on the constraint degree distribution pairs from Table I. Figure 1 shows the frame error rate (FER) versus $E_{\mathrm{b}} / N_{0}$ of $q$-ary PPM allowing a maximum of 50 decoding iterations. Observe that the waterfall performance is well predicted by the DE analysis. In addition, we provide the performance of three $R=1 / 2$ binary accumulaterepeat-4-jagged-accumulate (AR4JA) LDPC codes assuming a BICM setting and $q$-ary PPM for $q \in\{8,16,32\}$. The AR4JA protograph was taken from [11] and expanded to obtain block lengths (in bits) of $N \log _{2} q$. For $q \geq 16$ the performance of the non-binary codes under SMP decoding are competitive and for $q=32$ they outperform the AR4JA codes with BICM by almost $0.2 \mathrm{~dB}$.

\section{Complexity}

Despite the gap to capacity in Table I, SMP decoding might be a good choice when low-complexity decoding is targeted. First, a comparison of the algorithmic complexity of the SMP decoder (from Section III) and a binary LDPC decoder with BICM is given in Table II. For the binary decoder, the initialization step requires computing symbol-wise probabilities, followed by a marginalization to obtain bit-wise log-likelihood ratios (LLRs). The $\mathrm{CN}$ operations in the binary decoder follow the approximate min* rule [12]. The $\mathrm{VN}$ operations consist of summing up LLRs. Table II indicates that the algorithmic complexity of SMP is competitive with binary BP, but a fair comparison is difficult due to the different types of operations. E.g., the approximate min* rule and the SMP CN operations can be implemented by look-up tables. Then, an elementary 
TABLE I

Thresholds $\left(E_{\mathrm{b}} / N_{0}\right)^{\star}$ of $R=1 / 2$ LDPC COde EnSEMbles Under SMP. Shannon Limit $\left(E_{\mathrm{b}} / N_{0}\right)_{\text {Sh }}$ AS A REFERENCE. ENSEMbles With CONSTRAINTS ON LOW-DEGREE NODES ARE MARKED WITH $\bullet$.

\begin{tabular}{|c|c|c|c|c|c|}
\hline$q$ & $\lambda(x)$ & $\rho(x)$ & & $\left(E_{\mathrm{b}} / N_{0}\right)^{\star}[\mathrm{dB}]$ & $\left(E_{\mathrm{b}} / N_{0}\right)_{\mathrm{Sh}}[\mathrm{dB}]$ \\
\hline \multirow{2}{*}{4} & $0.0187 x+0.5597 x^{2}+0.003 x^{3}+0.4186 x^{11}$ & $0.3358 x^{7}+0.6642 x^{8}$ & & 3.22 & \multirow{2}{*}{1.06} \\
\hline & $0.3699 x^{2}+0.2799 x^{3}+0.3502 x^{11}$ & $0.0084 x^{7}+0.9916 x^{8}$ & - & 3.40 & \\
\hline \multirow{2}{*}{8} & $0.0465 x+0.5735 x^{2}+0.38 x^{11}$ & $0.859 x^{7}+0.141 x^{8}$ & & 2.19 & \multirow{2}{*}{0.08} \\
\hline & $0.4407 x^{2}+0.2652 x^{3}+0.2941 x^{11}$ & $0.5576 x^{7}+0.4424 x^{8}$ & - & 2.39 & \\
\hline \multirow{2}{*}{16} & $0.0595 x+0.5868 x^{2}+0.3537 x^{11}$ & $0.1351 x^{6}+0.8649 x^{7}$ & & 1.63 & \multirow{2}{*}{-0.47} \\
\hline & $0.5199 x^{2}+0.1436 x^{3}+0.3365 x^{11}$ & $0.5407 x^{7}+0.4593 x^{8}$ & - & 1.82 & \\
\hline \multirow{2}{*}{32} & $0.0582 x+0.6141 x^{2}+0.3277 x^{11}$ & $0.311 x^{6}+0.689 x^{7}$ & & 1.32 & \multirow{2}{*}{-0.80} \\
\hline & $0.55 x^{2}+0.1424 x^{3}+0.3076 x^{11}$ & $0.8045 x^{7}+0.1955 x^{8}$ & - & 1.48 & \\
\hline
\end{tabular}

TABLE II

COMPLEXITY SCALING OF ALGORITHMIC OPERATIONS.

\begin{tabular}{ccc}
\hline \hline & SMP & BP with BICM \\
\hline \multirow{2}{*}{ Init } & $\begin{array}{c}\mathcal{O}(N q) \\
\text { maximization }\end{array}$ & $\begin{array}{c}\mathcal{O}\left(N q \log _{2} q\right) \\
\text { sum of floats }\end{array}$ \\
\hline \multirow{2}{*}{$\mathrm{CN}$} & $\begin{array}{c}\mathcal{O}\left(M d_{\mathrm{c}} f(q)\right) \\
\text { elementary operations }\end{array}$ & $\begin{array}{c}\mathcal{O}\left(M d_{\mathrm{c}} \log _{2} q\right) \\
\text { box-plus } / \text { multiplications }\end{array}$ \\
\hline \multirow{2}{*}{$\mathrm{VN}$} & $\begin{array}{c}\mathcal{O}\left(N d_{\mathrm{v}}\right) \\
\text { addition of floats/maximization }\end{array}$ & $\begin{array}{c}\mathcal{O}\left(N d_{\mathrm{v}} \log _{2} q\right) \\
\text { addition of floats }\end{array}$ \\
\hline \hline
\end{tabular}

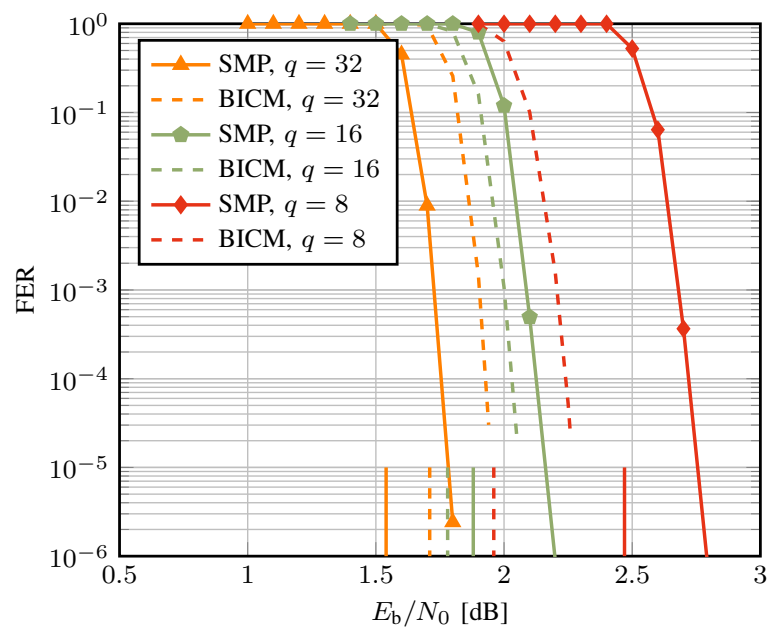

Fig. 1. FER versus $E_{\mathrm{b}} / N_{0}$ for SMP and BP with BICM. Respective thresholds are indicated by vertical lines.

operation is a look-up with $f(q)=1$ and complexity is reduced by a factor of $\log _{2} q$ w.r.t. the binary decoder. Other implementations may change the picture. Second, an important figure for implementation is the data flow in the decoder [13]. For SMP we need $\log _{2} q$ bits to represent a symbol, for binary BP typically 4 to 5 bits to represent an LLR. Since the binary Tanner graph has $\log _{2} q$ times more nodes the data flow of the SMP decoder will be lower by a factor of 4 to 5 (for the same average node degrees). Overall, the algorithmic complexity/data flow of an SMP decoder is highly competitive w.r.t. that of a binary decoder with BICM. However, only a hardware implementation will give final insights.

\section{CONCLUSIONS}

We investigate SMP decoding of $q$-ary LDPC codes on AWGN channels with $q$-ary orthogonal modulations. By showing that the extrinsic messages are observations of a $q \mathrm{SC}$ we apply DE to derive thresholds of unstructured code ensembles. A stability analysis suggests that not only degree two, but also degree three VNs impact stability. Thresholds of optimized irregular code ensembles reveal a gap of $2.1 \mathrm{~dB}$ w.r.t. the Shannon limit. Despite this gap, a comparison with binary LDPC codes and BICM shows performance gains for increasing $q$ with possible advantages in complexity of SMP. An open question is whether SMP decoders can be efficiently implemented in hardware.

\section{REFERENCES}

[1] M. Davey and D. MacKay, "Low density parity check codes over GF(q)," IEEE Communications Letters, vol. 2, pp. 70-71, 1996.

[2] Short block length LDPC codes for TC synchronization and channel coding, Orange Book, Issue 1, Consultative Committee for Space Data Systems (CCSDS) Experimental specification 231.1-O-1, Sep. 2015.

[3] D. Declercq and M. Fossorier, "Decoding algorithms for nonbinary LDPC codes over GF( $q$ )," IEEE Trans. Commun., vol. 55, no. 4, pp. 633-643, 2007

[4] V. Savin, "Min-max decoding for non binary LDPC codes," in Int. Symp. Inf. Theory, Toronto, Canada, Jul. 2008, pp. 960-964.

[5] E. Boutillon and L. Conde-Canencia, "Simplified check node processing in nonbinary LDPC decoders," in Int. Symp. Turbo Codes Iter. Inf. Proc., Brest, France, Sep. 2010, pp. 201-205.

[6] L. Barnault and D. Declercq, "Fast decoding algorithm for LDPC over GF $\left(2^{q}\right)$," in Inf. Theory Worksh., Paris, France, Mar. 2003, pp. 70-73.

[7] F. Lazaro, A. Graell i Amat, G. Liva, and B. Matuz, "Symbol message passing decoding of nonbinary low-density parity-check codes," in Global Commun. Conf., Waikoloa, HI, USA, Dec. 2019, pp. 1-5.

[8] A. Ashikhmin, G. Kramer, and S. ten Brink, "Extrinsic information transfer functions: Model and erasure channel properties," IEEE Trans. Inf. Theory, vol. 50, no. 11, pp. 2657-2673, 2004.

[9] G. Lechner, T. Pedersen, and G. Kramer, "Analysis and Design of Binary Message Passing Decoders," IEEE Trans. Commun., vol. 60, no. 3, pp. 601-607, Mar. 2012.

[10] G. Liva, B. Matuz, E. Paolini, and M. F. Flanagan, "Non-binary LDPC codes for orthogonal modulations: Analysis and code design," in IEEE Int. Conf. Commun., 2017, pp. 1-6.

[11] TM Synchronization and Channel Coding, Blue Book, Issue 2, Consultative Committee for Space Data Systems (CCSDS) Recommendation for Space Data System Standard 131.0.B.2, Aug. 2011.

[12] C. Jones, E. Valles, M. Smith, and J. Villasenor, "Approximate-min constraint node updating for LDPC code decoding," in Mil. Commun. Conf., vol. 1, Boston, MA, USA, May 2003, pp. 157-162.

[13] B. P. Smith, A. Farhood, A. Hunt, F. R. Kschischang, and J. Lodge, "Staircase Codes: FEC for $100 \mathrm{~Gb} / \mathrm{s}$ OTN," J. Lightw. Technol., vol. 30, no. 1, pp. 110-117, Jan. 2012 